\title{
SiGe EAM-Based Transceivers for Datacenter Interconnects and Radio over Fiber
}

\author{
Laurens Bogaert, Joris Van Kerrebrouck, Laurens Breyne, Joris Lambrecht, Haolin Li, Kasper Van Gasse, \\ Jochem Verbist, Michael Vanhoecke, Hannes Ramon, Srinivasan Ashwyn Srinivasan, Peter De Heyn, \\ Joris Van Campenhout, Peter Ossieur, Piet Demeester, Xin Yin, Johan Bauwelinck, Guy Torfs, \\ and Gunther Roelkens
}

(Invited Paper)

\begin{abstract}
Silicon photonics is a key-enabling technology leveraging decades of effort and infrastructure of the microelectronics CMOS industry resulting in high yield, low cost and potential high volume manufacturing. Furthermore, due to the high index contrast of the platform, very compact, high-complexity photonic integrated circuits can be devised. To benefit from these advantages, high-speed modulators should also be compatible with silicon technology. In this respect, SiGe electro-absorption modulators (EAM) are considered as a promising candidate since they are CMOS-compatible and offer high-speed, compact, low-loss and low-power modulation. In this paper, we discuss SiGe EAM-based transceivers for next-generation datacenter interconnects (DCI) and radio-over-fiber (RoF) fronthaul in nextgeneration cellular networks.
\end{abstract}

Index Terms-Data Center Interconnects (DCI), ElectroAbsorption Modulator (EAM), Integrated Optoelectronics, Radio-over-Fiber (RoF), Silicon Photonics (SiPh)

\section{INTRODUCTION}

$\mathbf{S}$ ILICON photonics is a key enabling technology which offers strong miniaturization of complex optical systems into an integrated circuit [1]-[4]. Potential applications are seemingly endless: (bio-)sensing, LIDAR, quantum computing, etc. Currently the main market for silicon photonics consists of transceivers for datacenters and long-haul optical communication. Furthermore, the market share of silicon photonics for $5 \mathrm{G}$ is also rapidly growing [5]. These silicon

Manuscript received August XX, 2020; revised January XX, 2020; accepted January XX, 2020. This work was supported by the Ghent University Special Research Fund (BOF14/GOA/034), the Methusalem funding of the Flemish government Grant Smart Photonic Chips, the European Research Council Grant ATTO (695495), and by the H2020 projects ICT-Streams (688172), Teraboard (688510), Picture (780930), and 5G-PHOS (761989). (Corresponding author: Laurens Bogaert)

L. Bogaert, L. Breyne, K. Van Gasse, and G. Roelkens are with the Department of Information Technology (INTEC), Photonics Research Group, Ghent University-imec, Ghent, 9052 Belgium (e-mail: \{laurens.bogaert; laurens.breyne; kasper.vangasse; gunther.roelkens\}@ugent.be).

J. Van Kerrebrouck, L. Breyne, J. Lambrecht, H. Li, M. Vanhoecke, H. Ramon, P. Ossieur, P. Demeester, X. Yin, J. Bauwelinck, and G. Torfs are with the Department of Information Technology (INTEC), IDLab, Ghent Universityimec, Ghent, 9052 Belgium (e-mail: \{joris.vankerrebrouck; laurens.breyne; joris.lambrecht; haolin.li; michael.vanhoecke; hannes.ramon; peter.ossieur; piet.demeester; xin.yin; johan.bauwelinck; guy.torfs\}@ugent.be).

J. Verbist is with BiFAST, Ghent, 9000 Belgium (e-mail: jochem@bifast.io)

S. A. Srinivasan, P. De Heyn, and J. Van Campenhout are with imec, Leuven, 3001 Belgium (e-mail: \{ashwyn.srinivasan; peter.deheyn; joris.vancampenhout\}@imec.be).

Color versions of one or more of the figures in this article are available online at https://ieeexplore.iee.org.

Digital Object Identifier XXX/JSTQE.XXX photonics integrated circuits rely on the infrastructure of the microelectronics CMOS industry to enable low-cost, highyield and high-volume manufacturing by implementing the optical functionality on a silicon-on-insulator (SOI) platform. Due to the high index contrast between silicon and its oxide, it is possible to realize complex optical functionality in a compact footprint. The silicon photonics platform used for the devices discussed in this paper is imec's iSiPP50G[6]. Apart from high-speed modulators and detectors, this platform also includes compact, low-loss waveguides, splitters, phase shifters, couplers, etc. For high-speed modulation [7], three types of components are available: carrier-depletion based Mach-Zehnder modulators (MZM), ring modulators and SiGe C-band electro-absorption modulators (EAM). In this paper, we focus on our circuits where EAMs [8] play a key role since this type of modulator is a promising candidate as it offers high-speed, low-power modulation on a compact footprint. Furthermore, EAMs are less wavelength-sensitive than ring modulators, both to changes in temperature and fabrication. Modulation in an EAM is based on the Franz-Keldysh effect where the bandgap can effectively be altered by applying an electric field. This will red shift the absorption curve and realizes intensity modulation for wavelengths near the bandgap. An increase in temperature narrows the bandgap resulting in a red shift of the absorption spectrum of the SiGe EAM by $0.8 \mathrm{~nm}$ per ${ }^{\circ} \mathrm{C}$ [6], [8]. However, the $1 \mathrm{~dB}$ optical bandwidth of the EAMs is approximately $30 \mathrm{~nm}$ [6] and therefore all but one of the experiments in this paper were performed without any temperature control. The only time temperature control was used, is for the frequency upconversion structure [30], where the TEC temperature was set to $20^{\circ} \mathrm{C}$.

This article consists of two main parts: transceivers for data center interconnects (DCI) applications (Sections II to IV) and Radio-over-Fiber (RoF) transceivers for mobile applications (Sections V to VIII). Section II demonstrates how 4level pulse-amplitude modulation (PAM-4) can be generated optically to avoid non-linearities in the modulator and allow for higher-efficiency non-linear drivers. Subsequently, Section III illustrates a 104 Gbaud optical time domain multiplexed (OTDM) transmitter multiplexing four data streams. Finally, Section IV shows that the EAMs can be used both for modulation and detection of an optical signal which is validated by implementing an EAM-based PAM-4 link.

The second part of this paper starts by describing a reflective 


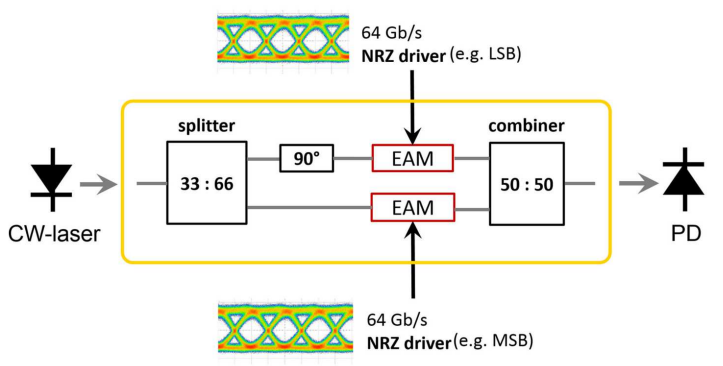

(a)

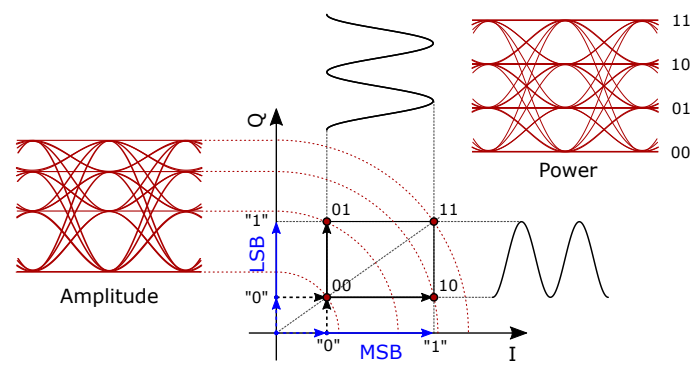

(b)

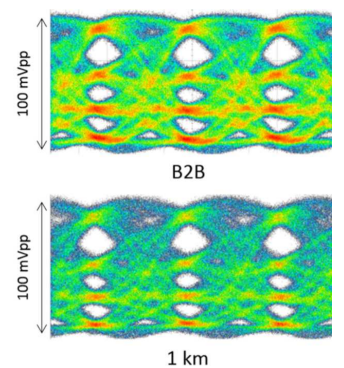

(c)

Fig. 1: 2-bit optical digital-to-analog converter (ODAC) for PAM-4 generation: (a) Operation principle; (b) Vector and eye diagrams; (c) Measured eye diagrams at 64 GBd in back-to-back (B2B) case and after 1 km SSMF

mmWave-over-Fiber transmitter in Section V. In Section VI, a technique is discussed to reduce the impractically high sampling rate of high carrier frequency Sigma-Delta-overFiber (SDoF) transmitters. Next, a quadrature EAM topology is discussed in Section VII where the required electrical $90^{\circ}$ phase shift is implemented optically. This structure is validated as an optical single sideband (OSSB) transmitter and as a full duplex transceiver. Finally, Section VIII covers two EAMbased architectures for photonic frequency conversion.

\section{OpticAl PAM-4 GENERATION}

Current standards for single mode DCI target 400 Gigbabit Ethernet $(\mathrm{GbE})$ by using 8 lanes of $53.125 \mathrm{~Gb} / \mathrm{s}$ PAM-4 for 1 and $2 \mathrm{~km}$ standard single mode fiber (SSMF) and 4 lanes of 106.25 Gb/s PAM-4 for 500m SSMF [9]. It is therefore clear that PAM-4 is the preferred modulation format for $400 \mathrm{GbE}$. However, multi-level formats bring additional complexity to the signal generation as they suffer from the non-linearities in the modulator and driver. Therefore, [10] discusses a technique to generate the PAM-4 signal optically using the structure shown in Fig. 1(a). The illustrated architecture enables the generation of PAM-4 using two EAMs connected in a MachZehnder interferometer (MZI) topology where each EAM is binary driven. Equidistant PAM-4 eyes can be generated using a 33:66 splitter at the input of the MZI while introducing an optical $90^{\circ}$ phase shift between both MZI arms. Because the EAMs are binary driven, more efficient drivers can be used than when linear PAM-4 drivers are required and the EAMs can be driven using stronger electrical input signals resulting in an increased extinction ratio ER (e.g. a dynamic ER of about $10 \mathrm{~dB}$ is obtained in [10] by driving the EAMs with $2.2 \mathrm{Vpp}$ and $1.1 \mathrm{Vpp}$ ). The resulting vector and eye diagrams of the proposed PAM-4 transmitter are shown in Fig. 1(b). Based on this principle, [10] demonstrated the generation of 64 Gbaud PAM-4 using two binary driven SiGe EAMs (Fig. 1(c)) capable of transmitting over B2B and $1 \mathrm{~km}$ SSMF with bit error rates (BERs) of 4E-10 and 8E-6 respectively, meeting the KP4-FEC limit (2.4E-4) without requiring electrical analog-todigital converters (ADCs), digital-to-analog converters (DACs) or digital signal processing (DSP). The described PAM-4 transmitter offers a lower power consumption and complexity compared to traditional PAM-4 transmitters where the
PAM-4 is generated electrically and fed to a single modulator, however, this comes at the cost of an added inherent $3 \mathrm{~dB}$ optical insertion loss compared to the single modulator. An example of an alternative ODAC solution is the segmented MZM [11] which typically results in a larger design but can offer increased ER or improved insertion loss compared to the proposed EAM-based solution which occupies $275 \mu \mathrm{m} \times$ $1720 \mu \mathrm{m}$ on the photonic IC.

\section{EAM-BASED OTDM}

Next generation DCI will target even higher data rates such as $800 \mathrm{~Gb} / \mathrm{s}$ and $1.6 \mathrm{~Tb} / \mathrm{s}$. This can be realized using more lanes, more complex data formats and/or higher baud rates. To preserve the number of lanes and avoid the increase in complexity and power consumption inherent to adopting PAM16, the symbol rate per lane should be increased. In this respect, 100 Gbaud will likely be the next standard symbol rate. However, such high lane rates require bandwidths of optical and electrical components to exceed approximately 70 GHz. Alternatively, [12] proposed to use optical time domain multiplexing (OTDM) to realize these higher baud rates. The concept of this scheme is shown in Fig. 2(a) and the fabricated device can be seen in Fig. 3. Such an OTDM structure allows for using lower-bandwidth electrical and optical components (quarter rate for a 4:1 serializer) but introduces an additional insertion loss ( $6 \mathrm{~dB}$ for a $4: 1$ serializer) and requires a pulsed optical source instead of the typically seen continuous wave laser at the input of the transmitter. Recent progress in modelocked lasers [13] makes this a solution to be considered. In [12], this optical 4:1 serializer was used to demonstrate the world's first intensity modulation, direct detection (IM/DD) silicon modulator capable of generating $208 \mathrm{~Gb} / \mathrm{s}(104 \mathrm{Gbaud}$ PAM-4) over a single wavelength, as shown in Fig. 2(b). Since each modulator is driven at a quarter of the symbol rate, which is 26 Gbaud in [12], required bandwidths of electrical and optical components are no longer challenging. Link experiments were carried out with this OTDM transmitter for 104 Gbaud on/off-keying (OOK), resulting in BER estimates of $7 \mathrm{E}-7$ (B2B) and 5E-6 (1 km SSMF) meeting KP4-FEC, and 104 Gbaud PAM-4, resulting in BER estimates of $8.9 \mathrm{E}-3$ (B2B) and 9.9E-3 (1km SSMF) meeting the soft decision FEC (2E-2) often employed for $200 \mathrm{~Gb} / \mathrm{s}$ coherent transceivers. 


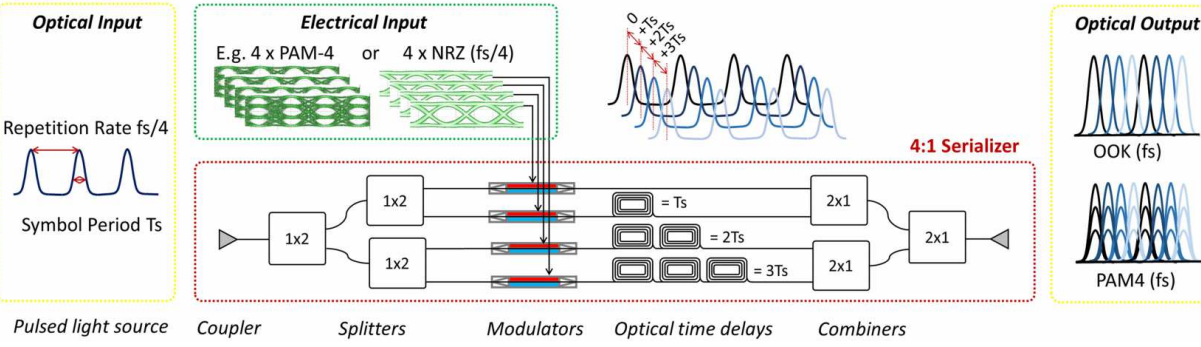

(a)

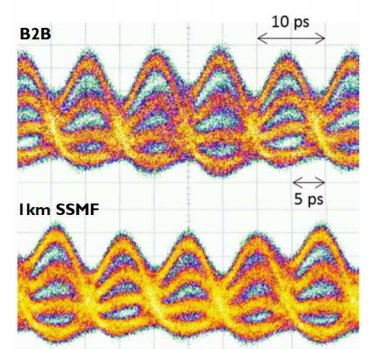

(b)

Fig. 2: Optical 4:1 serializer: (a) Operation principle; (b) Measured eye diagrams at $104 \mathrm{GBd}$ in B2B and after 1km SSMF

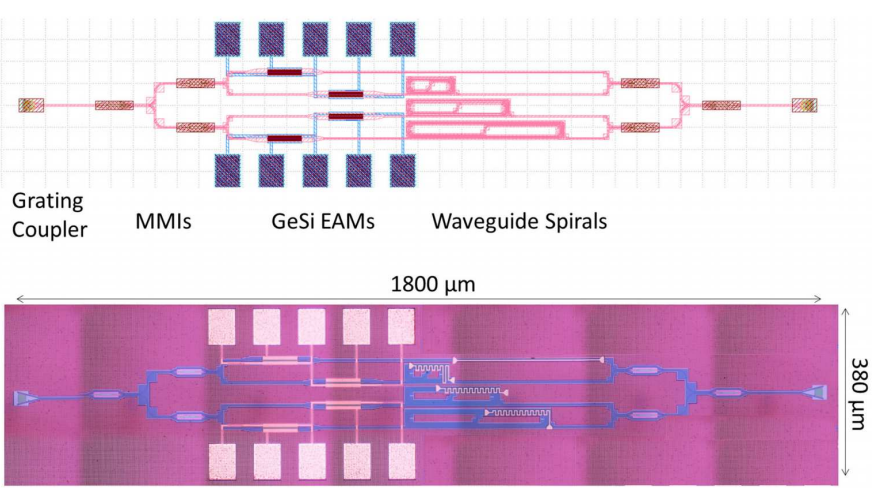

Fig. 3: Layout and microscope image of a 4:1 OTDM transmitter using EAMs

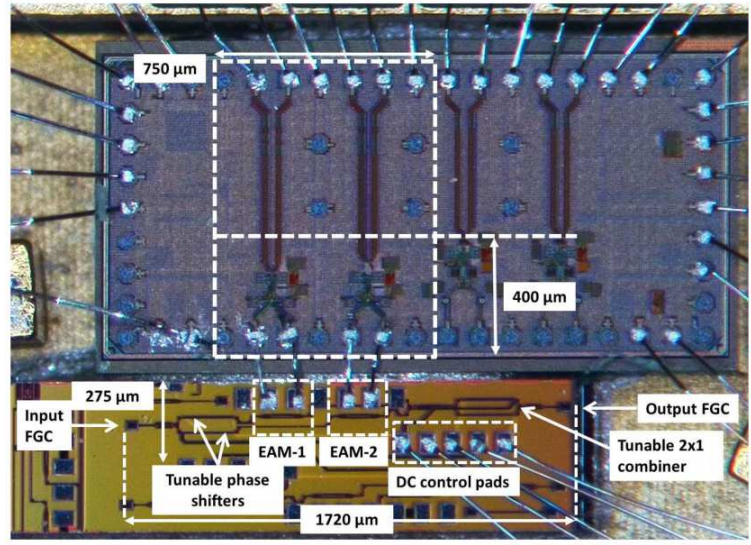

Fig. 4: Silicon integrated EAM PAM-4 transmitter.

For these experiments, PAM-4 was generated electrically and, apart from adjusting the placement of the PAM-4 levels in the arbitrary waveform generator (AWG) software, no DSP or equalization was used. The EAMs were driven with a $1.2 \mathrm{Vpp}$ electrical signal resulting in about $7 \mathrm{~dB}$ dynamic ER. Further improvements can be expected when PAM-4 is generated optically relying on the ODAC-principle discussed in the previous section, to mitigate the adverse affects caused by the non-linear E/O transfer function of the EAM.

\section{EAM-BASED PAM-4 LINK}

In previous sections, it was shown that EAMs can be a key building block in high speed optical transmitters. Meanwhile,

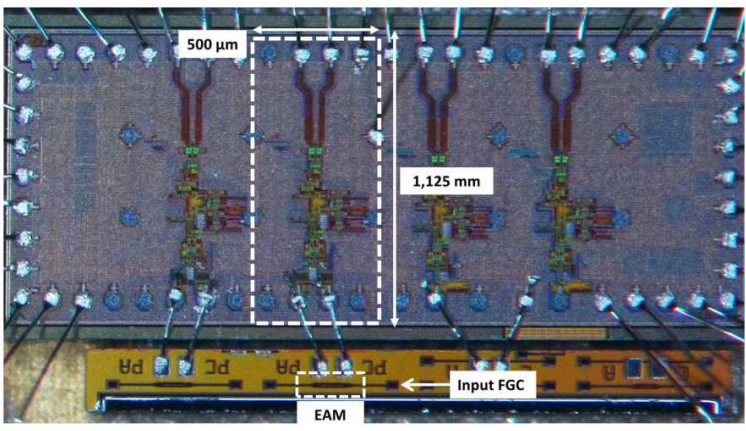

Fig. 5: Silicon integrated EAM PAM-4 receiver. the EAM also provides an output current proportional to the incident optical power. Therefore, the same EAM used to modulate the light can be used to perform detection at the receiver side. Adopting the same EAM for modulation and detection was explored in [10] and [14], where the transmitter (Fig. 4, [14]) and receiver (Fig. 5, [14]) were designed based on the same SiGe C-band EAM. At $1565 \mathrm{~nm}$ and $2.5 \mathrm{~V}$ reverse bias, the adopted EAM offers a responsivity of $0.725 \mathrm{~A} / \mathrm{W}$ and a $3 \mathrm{~dB}$ bandwidth over $50 \mathrm{GHz}$. The transmitter uses the ODAC principle discussed in Section II to generate 53 Gbaud PAM-4 using two $53 \mathrm{~Gb} / \mathrm{s} \mathrm{NRZ} \mathrm{streams} \mathrm{with} 300 \mathrm{mVpp}$ swing. As discussed in previous section, this ODAC transmitter results in an added $3 \mathrm{~dB}$ insertion loss but improves power efficiency of the transmitter and results in equidistant PAM-4 eyes. The ODAC was driven by a $55 \mathrm{~nm}$ SiGe BiCMOS driver IC [15] wirebonded to the modulators. With this transmitter, up to 53 Gbaud PAM-4 was transmitted over B2B, $1 \mathrm{~km}$ SSMF and $2 \mathrm{~km}$ dispersion shifted fiber (DSF) at $1565 \mathrm{~nm}$ with BER values of respectively $3.8 \mathrm{E}-6,7.9 \mathrm{E}-5$ and $2.6 \mathrm{E}-4$ [14]. The first two scenarios provide sub-KP4-FEC transmission and for the latter, the signal quality is just barely above KP-4 FEC but still well below 7\%-hard decision FEC (3.8E-3). At the receiver side, a standalone EAM was wirebonded to a $55 \mathrm{~nm}$ SiGe BiCMOS transimpedance amplifier IC [16]. With the aforementioned EAM-based transceiver, up to 40 Gbaud PAM4 was shown in [14] reaching BER values of 2.6E-5 (B2B) and 3.2E-3 (1 km SSMF), without any equalization or DSP resulting in a total power consumption of $310 \mathrm{~mW}(3.9 \mathrm{pJ} / \mathrm{b}$ at 40 Gbaud PAM-4), excluding the laser. 


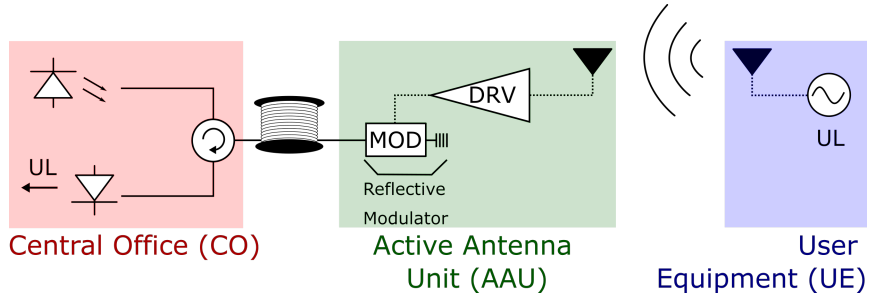

Fig. 6: Reflective implementation of the RFoF uplink path.

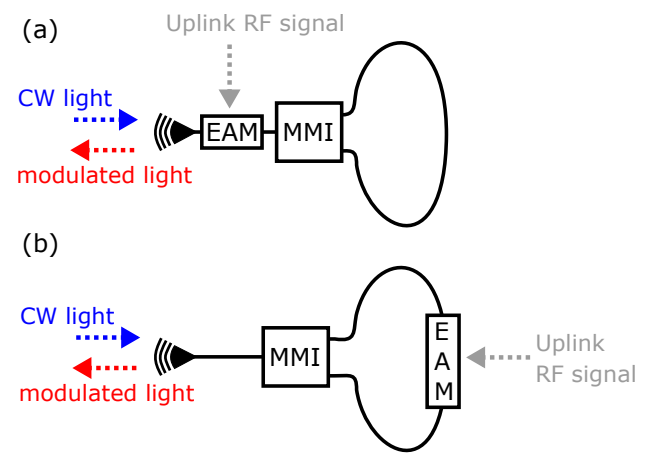

Fig. 7: Reflective EAM: (a) EAM in front of the loop mirror; (b) EAM embedded in the loop mirror.

\section{TX FOR LASER-LESS MMWAVE-OVER-FIBER UPLINK}

Densification of the communication cells and migration towards mmWave frequencies are two trends envisioned to cope with an ever-increasing demand for high-speed wireless data rates [17]. To make these techniques scalable, centralization is key and optical communication using the RoF principle [18] will be used to transfer the signal between the central office (CO) and the remote antenna units (RAUs). It is of paramount importance to make these RAUs as low-cost and low-complexity as possible. Hence, RF-over-Fiber (RFoF) is preferred over digitized RoF (DRoF, e.g. CPRI) or even IFover-Fiber as it avoids the need for the distribution of a synchronous carrier and does not require up/down-conversion or $\mathrm{ADC} / \mathrm{DAC}$ functionality at the RAU. To reduce the complexity and cost of the architecture even further, the uplink path should work in reflection (Fig. 6). This will make it possible to implement a single-fiber, circulator-less, laser-less RAU which will reduce cost and power consumption while relaxing stringent temperature control requirements at the RAU. For this purpose, a reflective EAM was designed to provide onchip reflective modulation where the mirror functionality is implemented using standard integrated silicon photonics.

\section{A. Reflective Electro-Absorption Modulator (R-EAM)}

The devised reflective modulator used in this paper comprises an EAM and a loop mirror [19]. This allows for an integrated reflective modulator constructed on the iSiPP50G silicon photonics platform. Alternatively, a bottom mirror can be added to a surface normal EAM [20] but this requires changing the platform and is thus to be avoided. A multimode interferometer (MMI) with one input and two outputs was used with interconnected outputs to construct the mirror. This can

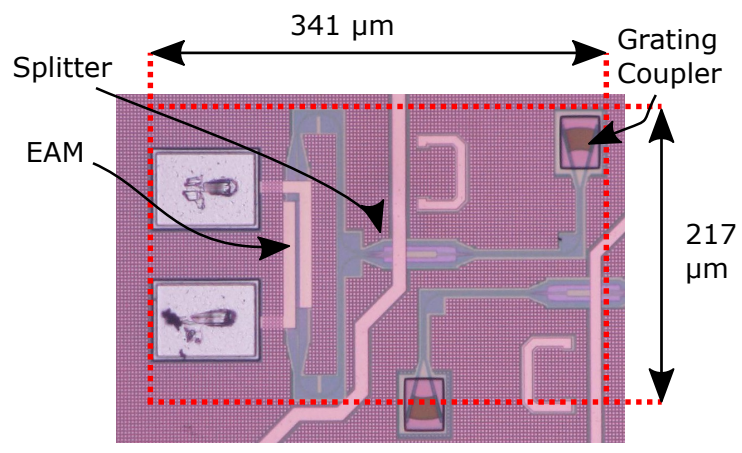

Fig. 8: Microscope image of the reflective EAM with modulator embedded in the loop mirror.

be done easily in silicon photonics due to the possibility to add bends with a small radius because of the high index contrast available in the SOI platform, resulting in a compact reflective modulator. The reflective EAM can then be implemented by either placing the EAM in front of the mirror (Fig. 7a) or by embedding it in the loop of the mirror (Fig. 7b). While the first will offer a larger modulation depth, it suffers from increased insertion losses. Due to challenges posed by back-reflection in the probing setup and in the fiber between $\mathrm{CO}$ and RAU, these increased on-chip insertion losses are to be avoided and the reflective modulator where the EAM is embedded inside the loop is preferred (Fig. 8).

The insertion loss of the reflective modulator consists of three loss contributions: I/O, modulator and mirror operation. First of all, the coupling from fiber to chip, which ideally results in $2.5 \mathrm{~dB}$ insertion loss for the adopted C-band grating couplers under optimal probing conditions but will typically be close to $5 \mathrm{~dB}$ in case of manual alignment. The grating coupler is passed twice (in and out) and thus we can expect approximately $10 \mathrm{~dB}$ insertion loss due to the coupling between fiber and chip. This contribution can however be improved significantly by using edge couplers and/or more accurate alignment. A second loss contribution is due to the modulator itself and will be dependent on the operation wavelength and bias voltage of the EAM. Assuming $1 \mathrm{~V}$ reverse bias at 1550 $\mathrm{nm}$, an insertion loss of $7 \mathrm{~dB}$ is found for a single EAM and if the EAM is placed in front of the mirror, this would be doubled due to double-passage of the light through the modulator. Finally, losses in the loop mirror itself are negligible.

For the assumed operation point, the R-EAM with the modulator embedded in the loop mirror thus results in approximately $17 \mathrm{~dB}$ fiber-to-fiber insertion loss, which can be improved to about $10 \mathrm{~dB}$ using an edge coupler instead of the grating coupler. Meanwhile, the unmodulated light fed to the R-EAM will partially be reflected at the flat glass/air interface of the fiber tip used to probe the grating coupler [21]. This reflection at the fiber tip results in a signal of comparable strength relative to the continuous wave $(\mathrm{CW})$ tone in the modulated light coming back from the R-EAM resulting in undesired interference effects. The back reflection can be heavily reduced by making use of index matching gel between the fiber tip and grating coupler and is therefore crucial in the correct operation of the devised reflective RFoF transmitter. 


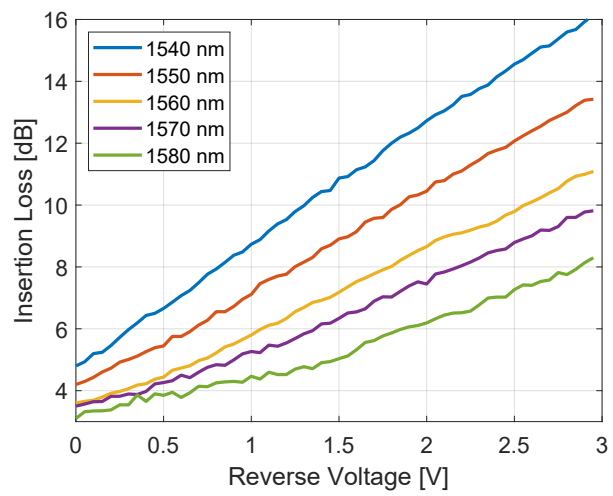

Fig. 9: Static insertion loss of the standalone $80 \mu \mathrm{m}$ EAMs.

\begin{tabular}{|r|l|}
\hline EAM bias & Impedance \\
\hline $0 \mathrm{~V}$ & $47.6-\mathrm{j} 44.9 \Omega$ \\
$1 \mathrm{~V}$ & $48.5-\mathrm{j} 63.1 \Omega$ \\
$2 \mathrm{~V}$ & $49.9-\mathrm{j} 68.9 \Omega$ \\
$3 \mathrm{~V}$ & $50.0-\mathrm{j} 73.7 \Omega$ \\
\hline
\end{tabular}

TABLE I: Impedance of the $80 \mu \mathrm{m}$ EAM at $28.5 \mathrm{GHz}$ including an additional $495 \mathrm{pH}$ wirebond inductance.

It should be noted that when such an index matching gel is present, the fiber angle used for probing the grating coupler should be changed accordingly.

The static insertion loss of the R-EAM depends on the operation wavelength and the adopted bias level. In Fig. 9, the insertion loss of a standalone EAM is plotted as a function of the applied bias voltage for multiple operation wavelengths. These values exclude any loss contributions caused by the grating couplers. It is clear that the insertion loss increases for stronger biasing of the EAM and for lower wavelengths.

\section{B. Driver \& R-EAM Transmitter}

A dedicated amplifier was designed to drive the modulator in the $28 \mathrm{GHz}$ band. The driver is implemented using a $0.1 \mu \mathrm{m}$ pHEMT GaAs technology and is based on the design of the low noise amplifier (LNA) in the narrowband photoreceiver discussed in [22]. Two major changes were, however, required to ensure that the LNA can drive an EAM. On the one hand, the input and output matching networks were readjusted. The original LNA in [22] expects a PD-wirebond combination at the input and $50 \Omega$ load at the output. For the EAM driver, these values change to a $50 \Omega$ at the input and a wirebondEAM combination at the output. On the other hand, the output matching network should include an internal bias tee to allow for easy biasing of the EAM.

The wirebond-EAM combination results in the load impedances provided in Table I. These values depend on the applied EAM bias and are given for a wirebond inductance of $495 \mathrm{pH}$. This inductance value was established in [22] by interpreting the wirebond pair as a current loop consisting of two conductors of $25 \mu \mathrm{m}$ diameter spaced $100 \mu \mathrm{m}$ apart and having a length of around $600 \mu \mathrm{m}$. The driver is designed for the value at $1 \mathrm{~V}$ EAM bias, namely $48.5-\mathrm{j} 63.1 \Omega$. The final layout of the driver is shown in Fig. 10, where the values next to the transmission line elements show changes in the matching network relative to the design of the LNA [22] to accommodate for different load and source impedances. Meanwhile, the core of the amplifier remained the same as in [22]. The assembled reflective narrowband transmitter is shown in Fig. 11 and comprises a $28 \mathrm{GHz}$ narrowband $\mathrm{GaAs}$ driver and reflective SiGe EAM, where the latter is constructed by embedding the EAM in a loop mirror. The power consumption depends on the drain supply of the amplifier and will be 124 $\mathrm{mW}$ for a $2 \mathrm{~V}$ supply and increases to $247 \mathrm{~mW}$ for a $3 \mathrm{~V}$ supply. In the remainder of this section, the small signal behavior, noise and linearity will be discussed in more detail.

The small signal measurements are performed in a $50 \Omega$ environment and the obtained S-parameters are subsequently transformed for the desired interface impedances, namely $50 \Omega$ at the input and a wirebond-EAM combination at the output. These transformed S-parameters of the driver are shown in Fig. 12(a)-(c) together with the simulated data for a $2 \mathrm{~V}$ driver supply and a 1V EAM bias, with no noticeable difference in small signal behavior when the driver supply is increased to $3 \mathrm{~V}$. The measured $S_{21}$ has a peak value of $25.7 \mathrm{~dB}$ at $26.8 \mathrm{GHz}$ and the $3 \mathrm{~dB}$ bandwidth ranges between 24.7 and $30.6 \mathrm{GHz}$. Furthermore, the $-10 \mathrm{~dB}$ return loss bandwidth covers 25.0 to $30.5 \mathrm{GHz}$ at the input and 26.5 to $30.4 \mathrm{GHz}$ at the output.

Subsequently, the transmitter was used in an RFoF link to characterize the transfer function of the entire transmitter including the electro-optic conversion in the modulator. A $70 \mathrm{GHz}$ photodetector was used as a receiver to make sure that bandwidth dependent effects of the link can be attributed to the transmitter. The adopted setup for these experiments is shown in Fig. 13 and a VOA was used to keep the DC power level incident on the photodetector constant. This allows us to ignore the wavelength dependent insertion losses and focuses on variations in modulation depth in the transmitter for different operation wavelengths. The results of these experiments are shown in Fig. 12(d) and it can be seen that there is a change in modulation depth when altering the operation wavelength. Nevertheless, the normalized transfer function appears to be independent of the wavelength. A decrease in bandwidth can be seen compared to the standalone driver. Whereas the 3-dB bandwidth of the driver ranges between 24.7 and $30.6 \mathrm{GHz}$, the transmitter results in a 3-dB bandwidth between 24.4 and $29.5 \mathrm{GHz}$ covering most of the new radio band $\mathrm{n} 258$ (24.25$27.5 \mathrm{GHz})$ and the entire $\mathrm{n} 257$ band $(26.5-29.5 \mathrm{GHz})$. This bandwidth reduction might be caused by an increased wirebond inductance and/or bandwidth limitations in the electrooptic conversion of the modulator.

Next, the noise of the devised RFoF transmitter should be quantified. Since the core design of the amplifier in [22] was reused, the noise figure (NF) is comparable. Simulated noise figure values can be found in Fig. 14 and the minimum NF for $2 \mathrm{~V}$ and $3 \mathrm{~V}$ driver supply is respectively 2.03 and $2.17 \mathrm{~dB}$.

To conclude the characterization, the linearity of the RFoF transmitter will be discussed. The adopted setup to characterize the input referred $1 \mathrm{~dB}$ compression point is shown in Fig. 15. The resulting values of the linearity at $28 \mathrm{GHz}$ are given in Table II for varying wavelengths and EAM bias levels given a $3 \mathrm{~V}$ driver supply and an optical power of $0 \mathrm{dBm}$ incident on the photodetector. Additional experiments show 


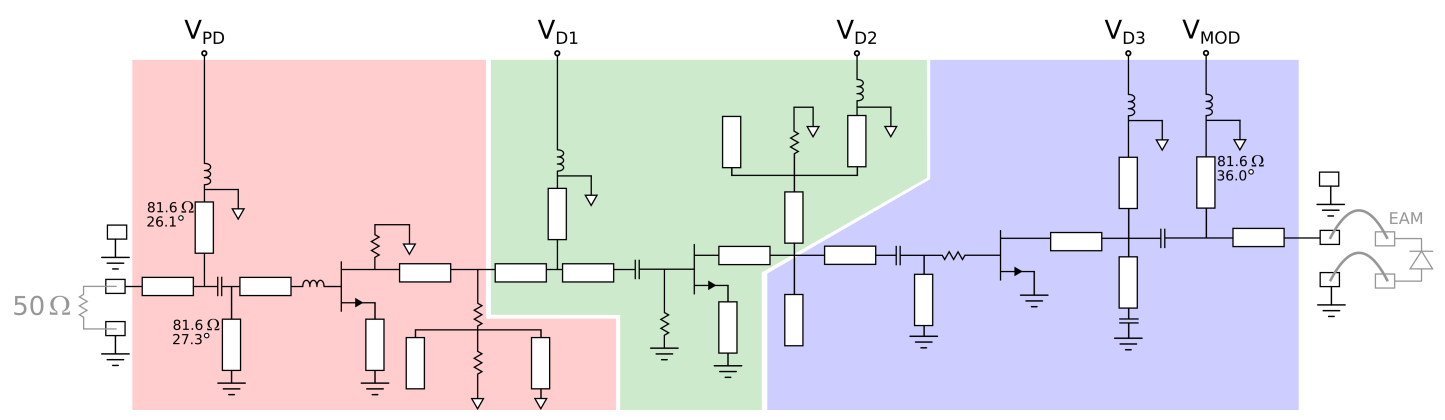

Fig. 10: Overall schematic of the 3-stage narrowband EAM driver.

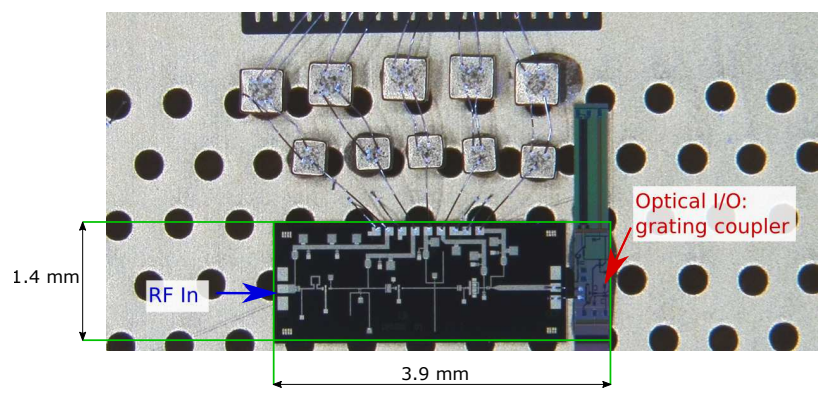

Fig. 11: Reflective $28 \mathrm{GHz}$ RFoF transmitter.

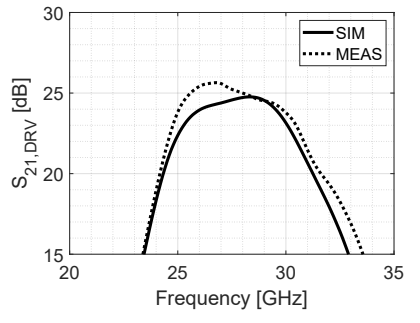

(a)

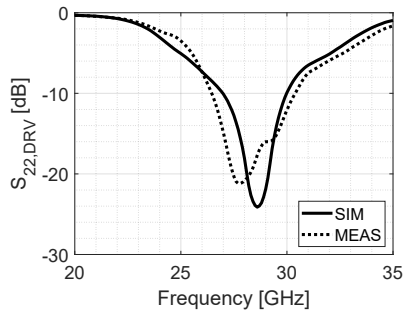

(c)

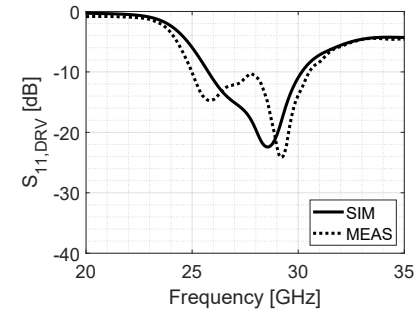

(b)

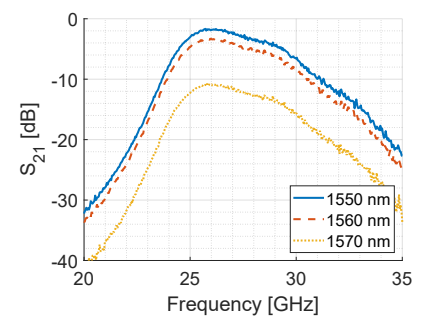

(d)
Fig. 12: (a)-(c) EAM driver S-parameters (d) Transfer function of the transmitter ( $2 \mathrm{~V}$ driver supply, $1 \mathrm{~V}$ EAM bias).

that the change in linearity is negligible when reducing the driver supply to $2 \mathrm{~V}$, showing that the modulator and not the driver determines the linearity of the transmitter. Furthermore, changing the optical power incident on the photodetector does not influence the linearity of the link, indicating that the transmitter is indeed the limiting factor of the linearity in the RFoF link. Based on table II, we can conclude that the linearity in the wavelength range of interest improves for increasing reverse bias of the EAM. However, stronger reverse biasing also increases insertion loss and results in increased reflections at the output of the driver.

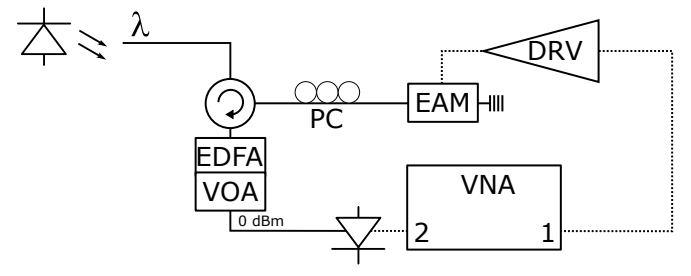

Fig. 13: Setup for $S_{21}$ characterization (PC: polarization controller; DRV: driver; VOA: variable optical attenuator).

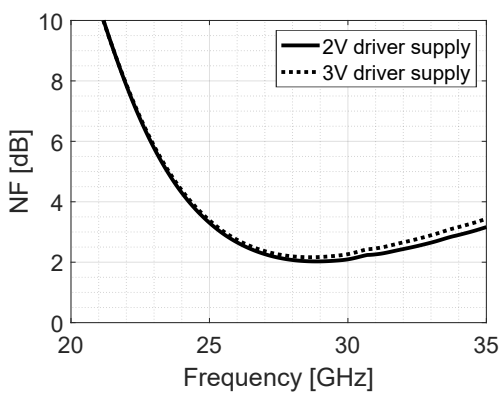

Fig. 14: Simulated noise figure of the RFoF transmitter.

\section{Link Experiments}

In [23], the RFoF transmitter discussed above was used in fiber-wireless link experiments together with the photoreceiver described in [22]. Due to the presence of a wavelength division multiplexer (WDM) in the adopted photoreceiver, the laser wavelength was fixed to $1550 \mathrm{~nm}$. Those experiments demonstrated a downlink and uplink data capacity of $12 \mathrm{~Gb} / \mathrm{s}$ and $8 \mathrm{~Gb} / \mathrm{s}$ for respectively $1 \mathrm{~m}$ (rms error vector magnitude EVM $<6.8 \%)$ and $3 \mathrm{~m}(\mathrm{rms} \mathrm{EVM}<9.7 \%)$ wireless distance in a mmWave-over-Fiber link with $2 \mathrm{~km}$ fiber meeting respectively the 3GPP criteria for 64-QAM (rms EVM $<8 \%$ ) and 16QAM (rms EVM $<12.5 \%$ ). For longer wireless distances, orthogonal frequency domain multiplexing (OFDM) operation is desired to overcome equalization challenges due to multipath induced channel fading. Using OFDM, up to $7.02 \mathrm{~Gb} / \mathrm{s}$ was achieved with an rms EVM below $10 \%$ over $2 \mathrm{~km}$ fiber and $5 \mathrm{~m}$ wireless distance with sufficient signal quality (rms EVM below $7.8 \%$ ) to allow for $10.53 \mathrm{~Gb} / \mathrm{s}$ operation when limiting the wireless distance to $1 \mathrm{~m}$. In these experiments, uplink and downlink operation were tested separately since new radio standards at $28 \mathrm{GHz}$ adopt a time division duplexing (TDD) scheme [24]. 


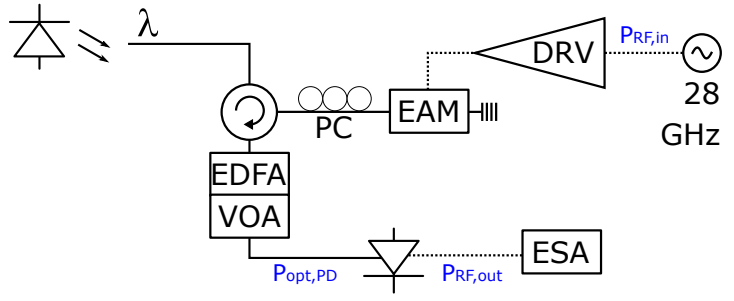

Fig. 15: Setup for $1 \mathrm{~dB}$ compression measurement (ESA: electrical spectrum analyzer).

\begin{tabular}{c|ccc} 
& $\lambda=\mathbf{1 5 5 0} \mathbf{~ n m}$ & $\lambda=\mathbf{1 5 6 0} \mathbf{~ n m}$ & $\lambda=\mathbf{1 5 7 0} \mathbf{~ n m}$ \\
\hline $\mathbf{V}_{\text {EAM }}=\mathbf{0 . 5 V}$ & $-26 \mathrm{dBm}$ & $-25 \mathrm{dBm}$ & $-23 \mathrm{dBm}$ \\
$\mathbf{V}_{\text {EAM }}=\mathbf{1 V}$ & $-24 \mathrm{dBm}$ & $-24 \mathrm{dBm}$ & $-22 \mathrm{dBm}$ \\
$\mathbf{V}_{\text {EAM }}=\mathbf{2 V}$ & $-20 \mathrm{dBm}$ & $-23 \mathrm{dBm}$ & $-20 \mathrm{dBm}$ \\
$\mathbf{V}_{\text {EAM }}=\mathbf{3 V}$ & $-18 \mathrm{dBm}$ & $-21 \mathrm{dBm}$ & $-16 \mathrm{dBm}$ \\
\hline
\end{tabular}

TABLE II: RFoF transmitter input referred $1 \mathrm{~dB}$ compression point at $28 \mathrm{GHz}$ (3V driver supply).

\section{SDOF EXPERIMENTS WITH EAM-TX}

In the previous section, RFoF was discussed as a technique to transfer data optically between $\mathrm{CO}$ and RAU. Three types of Radio-over-Fiber (RoF) are considered, as shown in Fig. 16, [25]. First, one can rely on digitized RoF (DRoF), offering good immunity to noise and non-linearities in the optical link. Unfortunately, this strategy results in complex RAUs and should therefore be avoided in high-frequency, small-cell mobile networks. Second, Analog RoF (ARoF), which includes RFoF, allows for simple RAUs, only requiring conversion between the optical and electrical domain in combination with amplification of the electrical signal. However, ARoF requires a low-noise, high-linearity link to achieve high signal quality. Sigma-Delta-over-Fiber (SDoF) combines the advantages of DRoF (high immunity to noise and non-linearities) and ARoF (low-complexity RAUs). The SDoF architecture starts from the baseband data, which is oversampled and quantized to a 1-bit signal [26]. Digital upconversion is then used to shift the signal to the correct RF carrier. At the receiver side of a SDoF link, the optical signal is converted back to an electrical signal, bandpass-filtered and amplified. Hence, such a SDoF optical link requires low-complexity receivers, while the immunity to noise and non-linearities is much higher than ARoF schemes since a binary signal is sent over the optical link.

Future mobile communication networks make use of very high carrier frequencies, resulting in impractically high required sampling rates of such SDoF transmitters. In that respect, a parallel EAM structure was used in [27] to modulate inphase (I) and quadrature (Q) components on separate EAMs and combine these two signals in the optical domain (Fig. 17, [27]). This helps to halve the required sampling rate of the Sigma-Delta transmitter. The resulting all-digital SDoF transmitter from [27], demonstrates up to $5.25 \mathrm{~Gb} / \mathrm{s}$ 64-QAM data transmission at $28 \mathrm{GHz}(\mathrm{n} 257: 26.5-29.5 \mathrm{GHz})$ over $10 \mathrm{~km}$ SSMF at $1560 \mathrm{~nm}$ with an rms EVM of $7.6 \%$ (this complies with the 3GPP requirement for 64-QAM, which states that the rms EVM should be below 8\%). Furthermore, by tuning the optical phase and the power combining ratio of the MZI, gain mismatch and chromatic dispersion notches can be alleviated.

\section{QUADRATURE EAM STRUCTURE}

In this section, the quadrature EAM structure, schematically shown in Fig. 18, is discussed. It consists of two EAMs where each modulator is located in a different arm of a MZI topology. Heaters, indicated by HTR, are present to thermally set the optical phase difference between both arms of the MZI and consequently set the bias point of the quadrature EAM structure. Importantly, the EAMs are found at different positions in both arms of the MZI. This is done to introduce an equivalent electrical delay between both modulators and should be designed depending on the required electrical phase difference that needs to be present between the signals fed to the two modulators. Migrating this RF delay to the optical domain helps to reduce insertion loss, size and complexity. An interesting device can be obtained when an equivalent quarter period delay $\left(90^{\circ}\right)$ at the RF frequency is realized between both modulators. In that scenario, the structure allows for optical single sideband (OSSB) generation and full duplex transceivers, as will be discussed in the remainder of this section. The licensed $28 \mathrm{GHz}$ band is of particular interest for future mobile communication and for that frequency, the required optical waveguide length difference to implement a quarter-period delay is $628.8 \mu \mathrm{m}$ (eq. 1). In equation $1, f_{R F}$ denotes the RF carrier frequency, $c$ the speed of light and $n_{g}$ the group index of the optical waveguide. In the iSiPP50G platform used to realize this quadrature EAM, the group index for a $450 \mathrm{~nm}$ wide strip waveguide is equal to 4.26 in C-band. The final design of this quadrature EAM structure targeting the $28 \mathrm{GHz}$ band is shown in Fig. 19.

$$
L=\frac{1}{4 f_{R F}} \frac{c}{n_{g}}
$$

\section{A. Optical Single Sideband Transmitter}

Compared to regular optical dual sideband communication, OSSB results in improved spectral efficiency and avoids chromatic dispersion notches. Optical single sideband generation for narrowband data is often implemented using optical filtering [28] or by quadrature driving the two inputs of a dualdrive Mach-Zehnder modulator [29]. By using the structure described above, OSSB generation is achieved based on the second principle but instead of constructing the quadrature variants of the signal electrically, both EAMs expect the same signal. The required relative $90^{\circ}$ phase shift between the arms, needed for OSSB, is implemented optically (Fig. 20).

Assume that the signal at the input of the EAMs is a cosine with a frequency of $28 \mathrm{GHz}$, which is the frequency where a quarter period delay is experienced between both EAMs. The amplitude modulator operation is then modelled by equation 2 where $m$ denotes the modulation depth while $A_{\text {out }}$ and $A_{\text {in }}$ respectively denote the amplitude of the EAM output and input optical field. Without loss of generality, these equations assume lossless modulation, as the actual loss will only influence the functional behavior when it differs in both arms. Together with these formulas, one can calculate the optical field at output 1 and 2 using equations 3 and 4 , where $\omega_{c}$ and $\phi_{c}$ are the frequency and phase of the optical carrier. 


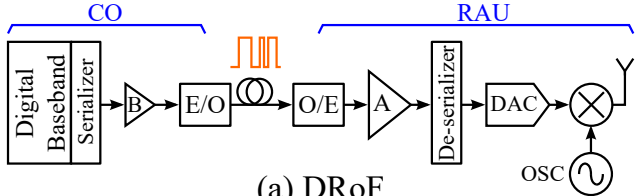

(a) DRoF

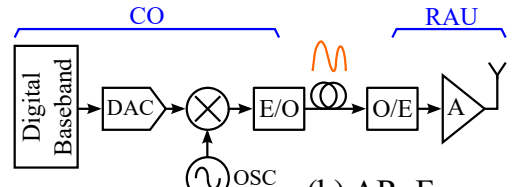

(b) ARoF

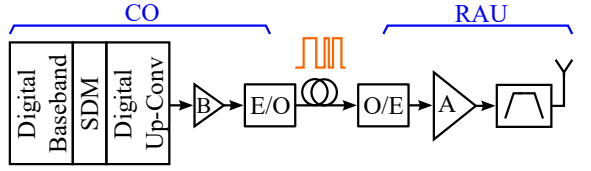

(c) $\mathrm{SDoF}$

Fig. 16: RoF implementation types - B: Binary Driver; A: Amplifier; OSC: Oscillator; SDM: Sigma Delta Modulator.
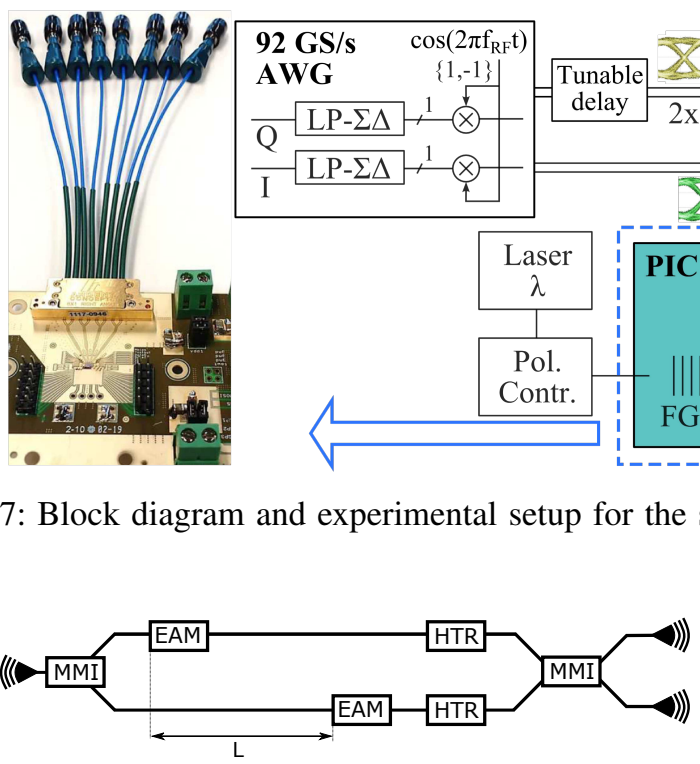

Fig. 18: Architecture of the quadrature EAM structure (HTR: heater; MMI: multi-mode interferometer).

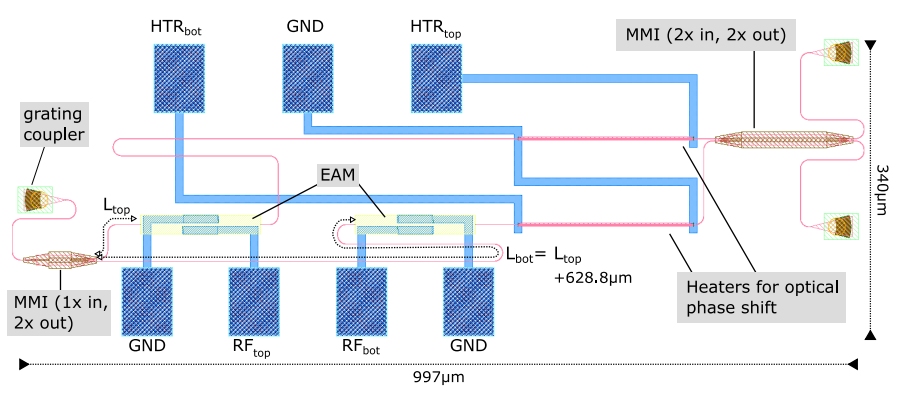

Fig. 19: Layout quadrature EAM for the $28 \mathrm{GHz}$ band

$$
\begin{aligned}
& \begin{cases}A_{\text {out }}(t) & =A_{\text {in }} \sqrt{(1-m)+m \cos \left(\omega_{R F} t\right)} \\
A_{\text {out }}\left(t-\frac{T}{4}\right) & =A_{\text {in }} \sqrt{(1-m)+m \sin \left(\omega_{R F} t\right)}\end{cases} \\
& E_{\text {out } 1}(t)=\frac{e^{-j \omega_{c} t} e^{-j \phi_{c}}}{2} \\
& \times\left[e^{j \pi / 2} A_{\text {out }}(t)+e^{j \phi_{\text {opt }}} A_{\text {out }}\left(t-\frac{T}{4}\right)\right] \\
& E_{\text {out } 2}(t)=\frac{e^{-j \omega_{c} t} e^{-j \phi_{c}}}{2} \\
& \times\left[A_{\text {out }}(t)+e^{j \phi_{\text {opt }}} e^{j \pi / 2} A_{\text {out }}\left(t-\frac{T}{4}\right)\right]
\end{aligned}
$$

Correct operation is verified using VPItransmissionMaker for three different settings of the optical phase $\phi_{\text {opt }}$, as shown

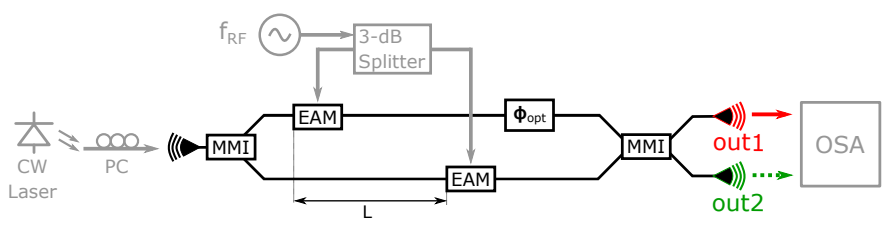

Fig. 20: OSSB generation with the quad-EAM structure (OSA: Optical spectrum analyzer).

in Fig. 21. When this optical phase shift equals $0^{\circ}$, OSSB signals are present both at output 1 and 2 respectively providing the lower and upper sideband. When a $180^{\circ}$ optical phase shift is applied, both outputs still present OSSB signals but now the upper and lower sideband variants are interchanged. Finally, it is interesting to take a closer look at what happens when a $90^{\circ}$ phase shift is set. This will result in a carrier-suppressed optical double sideband signal at output 2 . These results correspond with the behavior found when solving equations 3 and 4 .

To conclude the discussion of the OSSB transmitter, sideband suppression ratio (SBSR) degradation will be considered. Assume that the heater is set in such a way that $\phi_{\text {opt }}$ is exactly equal to $90^{\circ}$. It should be noted that non-perfect quadrature operation, i.e. the RF delay between the modulated signal in both arms is not exactly $90^{\circ}$, can have a significant influence. This deviation can be due to fabrication tolerances but it can also be introduced by having a finite signal bandwidth, i.e. narrowband approximation no longer holds, or by operating at carrier frequencies deviating from the design frequency. When the realized RF phase shift between the modulated signal in both arms is not exactly $90^{\circ}$, a finite SBSR is obtained, which is given in Fig. 22. A second hurdle to realize high SBSR relates to scenarios where the power in the top and bottom arm of the MZI differ. The influence of non-perfect 50/50 splitting in the input MMI is illustrated in Fig. 23. 

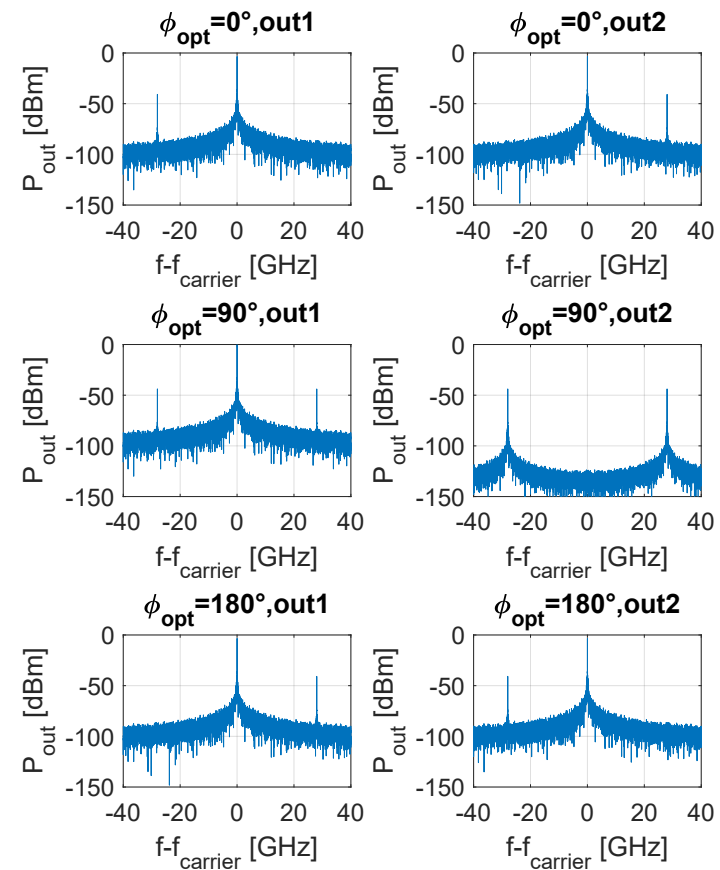

Fig. 21: Simulated output spectrum given $\phi_{\text {opt }}$ relative optical phase difference and equal RF input at both EAMs.

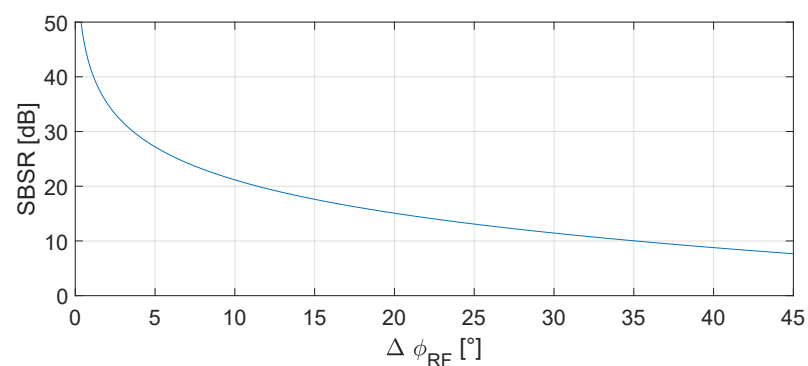

Fig. 22: Sideband suppression ratio (SBSR) as a function of deviation in the realized RF delay between the EAMs.

\section{B. Full Duplex Transceiver}

In a similar fashion as section IV, one can use the EAMs in this quadrature structure for simultaneous modulation and detection of RF signals. Together with an electrical $90^{\circ}$ hybrid coupler, this enables full duplex operation in the quadrature EAM with good isolation between downlink and uplink path. Such a quadrature electrical hybrid splits an input signal equally over both output ports with $90^{\circ}$ phase difference between both outputs. The full-duplex $28 \mathrm{GHz}$ transceiver is presented in Fig. 24 and it should be noted that output 1 is used as the output of the system and that $\phi_{\text {opt }}$ is set to $90^{\circ}$. First, consider uplink operation, i.e. the EAM modulates incoming CW laser light. The optical field presented at output OUTl can be written as eq. 5 which differs from eq. 3 because the signals applied to top and bottom EAM are no longer in phase. When a cosine with $28 \mathrm{GHz}$ RF frequency is applied to input port $R F 1$, the amplitude modulation in the top and bottom EAM correspond with eq. 6 resulting in a modulated signal at output port $O U T 1$. Furthermore, when that same

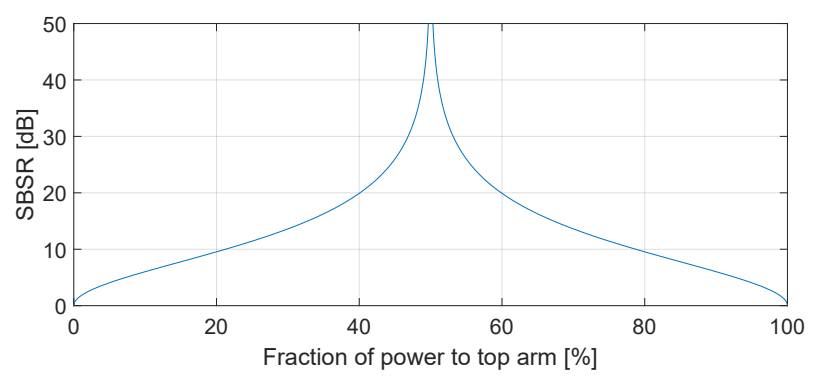

Fig. 23: Sideband suppression ratio (SBSR) as a function of power splitting ratio in the input MMI.

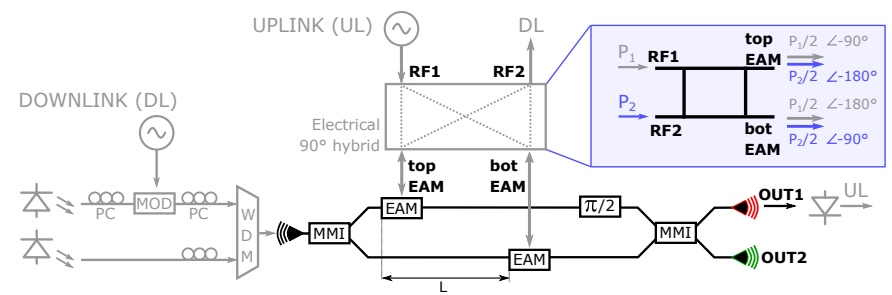

Fig. 24: Full duplex with the quad-EAM (MOD: Modulator, WDM: Wavelength division multiplexer; bot: Bottom).

cosine enters input port $R F 2$, the amplitude modulation in top and bottom EAM correspond with eq. 7 resulting in absence of modulation at output $O U T 1$. Hence, modulation occurs at $R F 1$ and modulation is isolated from any signal present at $R F 2$.

$$
\begin{aligned}
E_{\text {out } 1}(t) & =\frac{e^{-j \omega_{c} t} e^{-j \phi_{c}}}{2} \\
& \times\left[e^{j \pi / 2} A_{\text {out }, \text { bot }}(t)+e^{j \phi_{\text {opt }}} A_{\text {out }, \text { top }}\left(t-\frac{T}{4}\right)\right]
\end{aligned}
$$

$$
\begin{cases}A_{\text {out }, \text { bot }}(t) & =A_{\text {in }} \sqrt{(1-m)+m \cos \left(\omega_{R F} t-\pi / 2\right)} \\ A_{\text {out }, \text { top }}\left(t-\frac{T}{4}\right) & =A_{\text {in }} \sqrt{(1-m)+m \sin \left(\omega_{R F} t\right)}\end{cases}
$$

$$
\begin{cases}A_{\text {out }, \text { bot }}(t) & =A_{\text {in }} \sqrt{(1-m)+m \cos \left(\omega_{R F} t\right)} \\ A_{\text {out }, \text { top }}\left(t-\frac{T}{4}\right) & =A_{\text {in }} \sqrt{(1-m)+m \sin \left(\omega_{R F} t-\pi / 2\right)}\end{cases}
$$

To conclude this section, also the downlink needs to be considered. Assume light modulated at $28 \mathrm{GHz}$ enters the quadrature EAM, the output current of the top and bottom EAM will, amongst others, include a $28 \mathrm{GHz}$ cosine. After the modulated light enters the quadrature EAM, it is split and light needs to travel an extra $628.8 \mu \mathrm{m}$, corresponding to a quarter period at $28 \mathrm{GHz}$, to arrive at the bottom EAM relative to the top EAM. Consequently, for the downlink communication, the $28 \mathrm{GHz}$ cosine generated by the top EAM will lead by $90^{\circ}$ relative to the cosine generated by the bottom EAM. Both these currents generated by the EAMs are assumed to be equally strong and in that scenario, they will show perfect constructive interference at the $R F 2$ port of the electrical hybrid and perfect destructive interference at the $R F 1$ port. Consequently, the devised structure described in Fig. 24 offers 
an uplink RF input and downlink RF output, respectively at port $R F 1$ and $R F 2$ of the electrical hybrid, without needing an optical and/or electrical circulator. Furthermore, it can be seen that these two are perfectly isolated from each other. Isolation will degrade when the output current of both EAMs are not equally strong, when the electrical hybrid does not provide perfect 50/50 splitting, when the delay between both EAMs is not exactly a quarter-period of the RF carrier or when the electrical hybrid does not introduce proper $90^{\circ}$ phase difference between its outputs.

\section{FREQUENCY UPCONVERSION WITH EAM}

Next generation mobile networks target very high carrier frequencies as the higher end of the electromagnetic spectrum typically offers more contiguous bandwidth and is significantly less congested. Unfortunately, these high-carrier signals are not easily constructed in the electrical domain. Integrated microwave photonics can offer a solution by implementing the up- and downconversion optically such that the electrical data can be generated at a much lower intermediary frequency (IF) or even in baseband. Optical implementations of the mixing functionality allow for low-cost operation at a much higher frequency while offering significantly improved isolation and operation bandwidth. Two architectures for integrated photonic microwave frequency conversion have been presented by the authors in [30], [31]. In the first structure, a parallel EAM placed in a MZI topology was devised to realize the frequency conversion by feeding the IF signal to one of the EAMs while feeding the local oscillator (LO) signal to the other EAM as shown in Fig. 25, [30]. Heaters are present in the arms of the MZI to ensure that there is a $180^{\circ}$ phase shift between both arms. This will help to suppress the optical carrier in the output of the mixer. In [30], this photonic mixer was used to demonstrate upconversion of a $1.5 \mathrm{GHz}$ IF signal to arbitrary carrier frequencies in the range of 7 to $26 \mathrm{GHz}$. Furthermore, starting from an IF frequency of $3.5 \mathrm{GHz}$, [30] demonstrated upconversion to $28 \mathrm{GHz}$. After $2 \mathrm{~km}$ of SSMF, it was shown that 218.75 Mbaud, 16-QAM generated with this mixer, was received with an rms-EVM of $8.3 \%$, meeting the 3GPP NR specifications for 16-QAM [32]. This mixer offers a conversion loss of approximately $20 \mathrm{~dB}$ given a $1.5 \mathrm{GHz}$ IF frequency and $24 \mathrm{GHz}$ RF frequency. Furthermore it was established that this mixer offers a spurious free dynamic range (SFDR) of $82 \mathrm{~dB} \cdot \mathrm{Hz}^{2 / 3}$.

A second, more flexible, photonic mixer has been discussed in [31]. This mixer is depicted in Fig. 26, [31] and expects a dual tone laser light at its input. The frequency separation of this dual tone input should be equal to the LO frequency, in case of upconversion from the IF signal, or RF frequency, when starting from I/Q baseband data. The two laser lines are deinterleaved using microring resonators. Subsequently the light present at the drop port of the microring is modulated. Depending on the splitting ratio of the tunable splitters, the dropped tone is modulated by an EAM-based MZM, a single EAM or even a combination of both. In [31], only the first scenario is considered. The modulated and unmodulated laser tones are subsequently recombined by the second microring

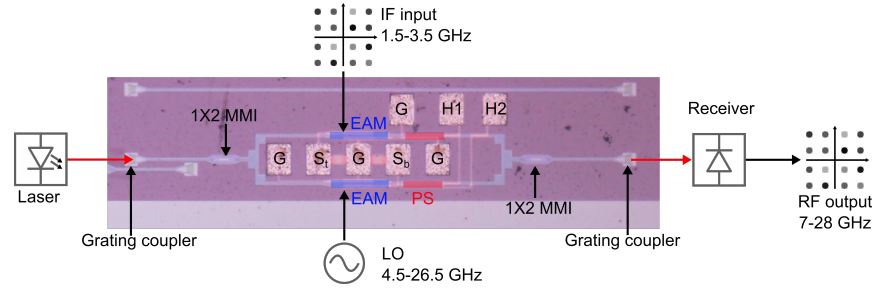

Fig. 25: Parallel EAM structure for upconversion.

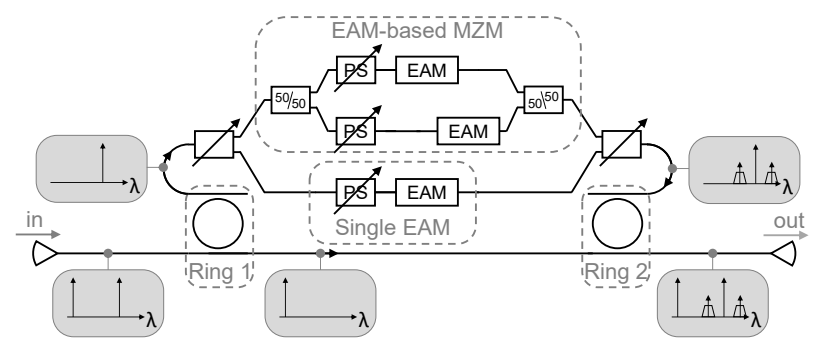

Fig. 26: Microring-assisted upconversion (PS: Phase shifter).

resonator resulting in the output light containing the upconverted data. As discussed, the modulating path can be routed to a single EAM which only provides amplitude modulation. The modulating path can also be switched to the MZM-EAM with $90^{\circ}$ optical phase shift between both arms to enable coherent modulation and as such making upconversion of baseband data possible. In [31], the modulating path was configured to pass all the light through the EAM-based MZM. An IF signal was modulated on both arms with an electrical phase shift of $90^{\circ}$ between both arms to construct an OSSB signal. Using this photonic mixer and starting from an IF frequency of 3.5 $\mathrm{GHz}$, [31] demonstrated upconversion to the $24-28 \mathrm{GHz}$ band which includes the $\mathrm{n} 258$ band ranging from 24.25 to 27.5 GHz. 50 Mbaud 64-QAM, or 200 Mbaud 16-QAM can be generated with an rms-EVM of respectively $3.6 \%$ and $10.4 \%$, meeting the 3GPP NR specifications [32]. This mixer offers a conversion loss of approximately $26 \mathrm{~dB}$ given a $3.5 \mathrm{GHz} \mathrm{IF}$ frequency and $24 \mathrm{GHz}$ RF frequency.

\section{CONCLUSION}

Interconnects in data centers and in the fronthaul of cellular networks require increasingly faster data rates and to accomplish this, optical communication is the key. In this paper, silicon photonics solutions for these applications are covered since silicon photonics is an integrated platform leveraging the microelectronics CMOS industry and its benefits such as low cost, high yield and high volume capabilities. Silicon photonics is a high index contrast integrated photonics platform and can thus realize high complexity devices on small chip areas. In this work, we have specifically looked at devices consisting of SiGe electro-absorption modulators. These are compact devices that offer low-power, high-speed modulation. In the first part of this paper, we showcased how these EAMs can result in high-speed, low-power DCI transmitters and even receivers. In the second part, we discussed several structures that can play a key role in the RoF fronthaul for nextgeneration mobile networks. 


\section{REFERENCES}

[1] B. Jalali, and S. Fathpour, "Silicon Photonics," J. Light. Technol., vol. 24, no. 12, pp. 4600-4615, Dec. 2006.

[2] X. Chen, M. M. Milosevic, S. Stankovic, S. Reynolds, T. D. Bucio, K. Li, D. J. Thomson, F. Gardes, and G. T. Reed, "The Emergence of Silicon Photonics as a Flexible Technology Platform," Proc. IEEE, vol. 106, no. 12, pp. 2101-2116, Dec. 2018.

[3] C. R. Doerr, "Silicon photonic integration in telecommunications," Front. Phys., vol. 3, Aug. 2015.

[4] J. W. Silverstone, D. Bonneau, J. L. O'Brien, and M. G. Thompson, "Silicon Quantum Photonics," IEEE J. Sel. Top. Quantum Electron., vol. 22, no. 6, Nov./Dec. 2016.

[5] Yole Développement, "Silicon Photonics - Market and Technology Report 2020," Apr. 2020.

[6] M. Pantouvaki, S. A. Srinivasan, Y. Ban, P. De Heyn, P. Verheyen, G. Lepage, H. Chen, J. De Coster, N. Golshani, S. Balakrishnan, P. Absil, and J. Van Campenhout, "Active Components for $50 \mathrm{~Gb} / \mathrm{s}$ NRZ-OOK Optical Interconnects in a Silicon Photonics Platform," J. Light. Technol., vol. 35, no. 4, pp. 631-638, Feb. 2017.

[7] J. Witzens, "High-Speed Silicon Photonics Modulators," Proc. IEEE, vol. 106, no. 12, pp. 2158-2182, Dec. 2018.

[8] S. A. Srinivasan, M. Pantouvaki, S. Gupta, H. T. Chen, P. Verheyen, G. Lepage, G. Roelkens, K. Saraswat, D. Van Thourhout, P. Absil, and J. Van Campenhout, " $56 \mathrm{~Gb} / \mathrm{s}$ Germanium Waveguide ElectroAbsorption Modulator," J. Light. Technol., vol. 34, no. 2, pp. 419-424, Jan. 2016.

[9] IEEE P802.3bs 400 Gigabit Ethernet Task Force, July 2020. [Online]. Available: http://www.ieee802.org/3/bs/

[10] J. Verbist, J. Lambrecht, M. Verplaetse, S. A. Srinivasan, P. De Heyn, T. De Keulenaer, R. Pierco, A. Vyncke, J. Van Campenhout, X. Yin, J. Bauwelinck, G. Torfs, and G. Roelkens, "Real-Time and DSPFree $128 \mathrm{~Gb} / \mathrm{s}$ PAM-4 Link Using a Binary Driven Silicon Photonic Transmitter," J. Light. Technol., vol. 37, no. 2, pp. 274-280, Jan. 2019.

[11] A. Samani, D. Patel, M. Chagnon, E. El-Fiky, R. Li, M. Jacques, N. Abadia, V. Veerasubramanian, and D. V. Plant, "Experimental parametric study of $128 \mathrm{~Gb} / \mathrm{s}$ PAM-4 transmission system using a multi-electrode silicon photonic Mach Zehnder modulator," Opt. Express, vol. 25, no. 12, pp. 13252-13262, Jun. 2017.

[12] J. Verbist, M. Vanhoecke, M. Lillieholm, S. A. Srinivasan, P. De Heyn, J. Van Campenhout, M. Galili, L. K. Oxenløwe, X. Yin, J. Bauwelinck, and G. Roelkens, "4:1 Silicon Photonic Serializer for Data Center Interconnects Demonstrating 104 Gbaud OOK and PAM4 Transmission," J. Light. Technol., vol. 37, no. 5, pp. 1498-1503, March 2019.

[13] Z. Wang, K. Van Gasse, V. Moskalenko, S. Latkowski, E. Bente, B. Kuyken, and G. Roelkens, "A III-V-on-Si ultra-dense comb laser," Light Sci. Appl., vol. 6, no. 5, May 2017.

[14] J. Lambrecht, J. Verbist, H. Ramon, M. Vanhoecke, J. Bauwelinck, $\mathrm{X}$. Yin, and G. Roelkens, "Low-Power (1.5 pJ/b) Silicon Integrated 106 Gb/s PAM-4 Optical Transmitter," J. Light. Technol., vol. 38, no. 2, pp. 432-438, Jan. 2020.

[15] H. Ramon, J. Lambrecht, J. Verbist, M. Vanhoecke, S. A. Srinivasan, P. De Heyn, J. Van Campenhout, P. Ossieur, X. Yin, and J. Bauwelinck, "70 Gb/s Low-Power DC-Coupled NRZ Differential Electro-Absorption Modulator Driver in $55 \mathrm{~nm}$ SiGe BiCMOS," J. Light. Technol., vol. 37, no. 5, pp. 1504-1514, March 2019.

[16] J. Lambrecht, H. Ramon, B. Moeneclaey, J. Verbist, M. Verplaetse, M. Vanhoecke, P. Ossieur, P. De Heyn, J. Van Campenhout, J. Bauwelinck, and X. Yin, "90-Gb/s NRZ Optical Receiver in Silicon Using a Fully Differential Transimpedance Amplifier," J. Light. Technol., vol. 38 , no. 2 , pp. 432-438, Jan. 2020.

[17] J. G. Andrews, S. Buzzi, W. Choi, S. V. Hanly, A. Lozano, A. C. K. Soong, and J. C. Zhang, "What Will 5G Be?," IEEE J. Sel. Areas Commun., vol. 32, no. 6, pp. 1065-1082, June 2014.

[18] C. Lim, A. Nirmalathas, M. Bakaul, P. Gamage, K. Lee, Y. Yang, D. Novak, and R. Waterhouse, "Fiber-Wireless Networks and Subsystem Technologies," J. Light. Technol., vol. 28, no. 4, pp. 390-405, Feb. 2010.

[19] F. Soltani, M. Menard and A. G. Kirk, "Integrated silicon photonic reflective modulator for passive optical networks," in Conference on Lasers and Electro-Optics, 2017, Paper JW2A.127.

[20] S. Grillanda, T.-C. Hu, D. Neilson, N. Basavanhally, Y. Low, H. Safar, M. Cappuzzo, R. Kopf, A. Tate, G. Raybon, A. Adamiecki, N. Fontaine, and M. Earnshaw, "107 Gb/s Ultra-High Speed, Surface-Normal Electroabsorption Modulator Devices," J. Light. Technol., vol. 38, no. 4, pp. 804-810, Feb. 2020.

[21] B. E. A. Saleh, and M. C. Teich , "Fundamentals of Photonics," 1st ed., Wiley, p. 209, 1991.
[22] L. Bogaert, H. Li, K. Van Gasse, J. Van Kerrebrouck, J. Bauwelinck, G. Roelkens, and G. Torfs, "36 Gb/s Narrowband Photoreceiver for mmWave Analog Radio-over-Fiber," J. Light. Technol., vol. 38, no. 12, pp. 3289-3295, June 2020.

[23] L. Bogaert, J. Van Kerrebrouck, H. Li, I. L. de Paula, K. Van Gasse, S. Lemey, H. Rogier, P. Demeester, G. Roelkens, J. Bauwelinck, and G. Torfs, "SiPhotonics/GaAs 28-GHz Transceiver for mmWave-overFiber Laser-Less Active Antenna Units," in Proc. Opt. Fiber Commun. Conf., 2020, Paper Th4A.5.

[24] 3GPP, "TR 38.815: New frequency range for NR $(24.25-29.5 \mathrm{GHz})$ - Rel. 15, v15.0.0," Jun. 2018, [Online]. https://www.3gpp.org/dynareport/38-series.htm

[25] C.-Y. Wu, H. Li, O. Caytan, J. Van Kerrebrouck, L. Breyne, J. Bauwelinck, P. Demeester, and G. Torfs, "Distributed Multi-User MIMO Transmission Using Real-Time Sigma-Delta-Over-Fiber for Next Generation Fronthaul Interface," J. Light. Technol., vol. 38, no. 4, pp. 705-713, Feb. 2020

[26] H. Li, L. Breyne, J. Van Kerrebrouck, M. Verplaetse, C.-Y. Wu, P. Demeester, and G. Torfs, "A 21-GS/s Single-Bit Second-Order Delta-Sigma Modulator for FPGAs," IEEE Trans. Circuits Syst., II, Exp. Briefs, vol. 66, no. 3, pp. 482-486, March 2019.

[27] H. Li, J. Van Kerrebrouck, H. Ramon, L. Bogaert, J. Lambrecht, C.Y. Wu, L. Breyne, J. Declercq, J. Bauwelinck, X. Yin, P. Ossieur, P. Demeester, and G. Torfs, "Low Power All-Digital Radio-over-Fiber Transmission for 28-GHz Band using Parallel Electro-Absorption Modulators," in Proc. Opt. Fiber Commun. Conf., 2020, Paper M2F.6.

[28] S. R. Blais, and J. Yao, "Optical Single Sideband Modulation Using an Ultranarrow Dual-Transmission-Band Fiber Bragg Grating," IEEE Photon. Technol. Lett., vol. 18, no. 21, pp. 2230-2232, Nov 2006.

[29] C. W. Chow, C. H. Wang, C. H. Yeh, and S. Chi, "Analysis of the carrier-suppressed singlesideband modulators used to mitigate Rayleigh backscattering in carrier-distributed PON," Opt. Express, vol. 19, no. 11, pp. 10973-10978, May 2011.

[30] K. Van Gasse, J. Verbist, H. Li, G. Torfs, J. Bauwelinck, and G. Roelkens, "Silicon Photonics Radio-Over-Fiber Transmitter Using GeSi EAMs for Frequency Up-Conversion," IEEE Photon. Technol. Lett., vol. 31, no. 2, pp. 181-184, Jan. 2019.

[31] L. Breyne, J. Verbist, J. Zhang, P. Demeester, G. Roelkens, and G. Torfs, "Flexible Integrated Silicon Photonic mm-Wave Frequency Upconversion Using GeSi EAMs," in Proc. Eur. Conf. Integr. Opt., 2019.

[32] 3GPP, "TR 36.104: LTE; Evolved Universal TerrestrialRadio Access; Base Station radio transmission and reception - Rel. 15, v. 15.3.0," Jul. 2018, [Online]. https://www.3gpp.org/dynareport/36-series.htm

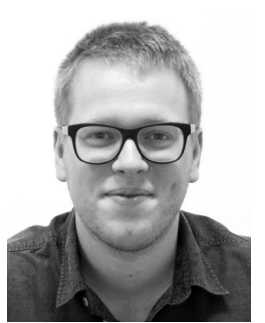

Laurens Bogaert received a $\mathrm{PhD}$ in electrical engineering from the Department of Information Technology (INTEC), Ghent University-imec, Belgium in 2020 and a M.Sc. degree in electrical engineering from Ghent University, Belgium in 2015. From 2020 on, he is working as a postdoctoral researcher at the Photonics Research Group, INTEC, Ghent University-imec, Belgium. His current research interests include III/V-on-Silicon transfer printing and opto-electronic co-integration for radioover-fiber links.

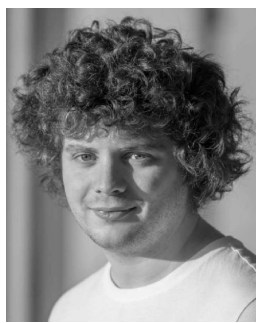

Joris Van Kerrebrouck was born in Ghent, Belgium, in 1989. He received the M.Sc. and Ph.D. degrees in electrical engineering from Ghent University, Ghent, Belgium, in 2014 and 2020, respectively. $\mathrm{He}$ is currently a postdoctoral researcher at IDLab Design Group, Department of Information Technology (INTEC), Ghent University. His research interests include radio over fiber, point-to-point optical links, and nonlinear electro-optical systems. 


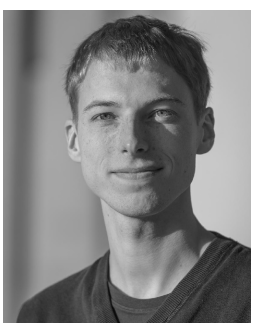

Laurens Breyne received the B.Sc. and M.Sc. degrees in electrical engineering from Ghent University, Ghent, Belgium, in 2014 and 2016, respectively, where he is currently pursuing the Ph.D. degree. In 2016, he joined IDLab Design Group, Ghent University-imec, Ghent. His main research interests include electrooptic codesign, high-speed optical interconnects, and high-speed analog design.

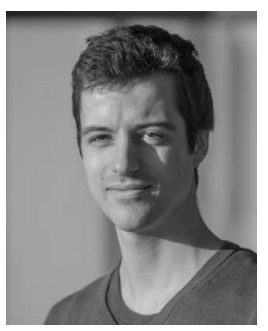

Joris Lambrecht received the M.Sc. and Ph.D. degrees in electrical engineering from Ghent University, Ghent, Belgium, in 2015 and 2019, respectively. $\mathrm{He}$ is currently working at imec, Ghent, with a focus on optical transceivers and high-speed optical receivers.

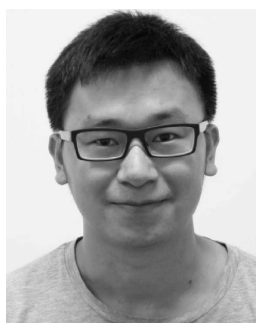

Haolin Li received the M.Sc. and Ph.D. degrees in electrical engineering from Ghent University, Ghent, Belgium, in 2014 and 2019, respectively. $\mathrm{He}$ is currently a postdoctoral researcher at IDLab Research Group, Department of Information Technology (INTEC), Ghent University. His research interests include signal processing and digital circuit design for wireless communication and radio-overfiber technology.

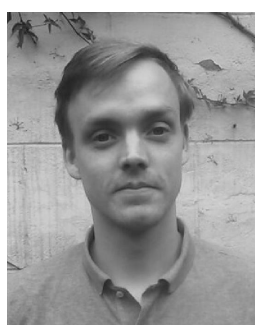

Kasper Van Gasse received a $\mathrm{PhD}$ in the Photonics Research Group at Ghent University-imec in 2019 and a MSc in Engineering Physics at Ghent University in 2014. He currently holds a Junior postdoctoral fellowship of the FWO. During this fellowship he is working towards the development of novel integrated mode-locked lasers for microwave photonics and spectroscopy.

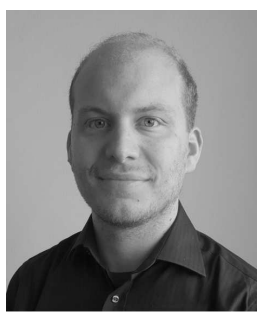

Jochem Verbist received his M.Sc. in electrical engineering from Ghent University in 2013. From 2014, he is pursuing a Ph.D. degree in a joint research activity between the Photonics Research Group and IDLab's high speed design group, both at Ghent University-imec. His research interests include high-speed optical interconnects, electrooptical design, high speed analog electronics, and integrated photonics. Currently, he is working at $\mathrm{Bi}$ FAST (Ghent, Belgium) on the development of $400 \mathrm{G}$ and $800 \mathrm{G}$ transceivers for data center interconnects.

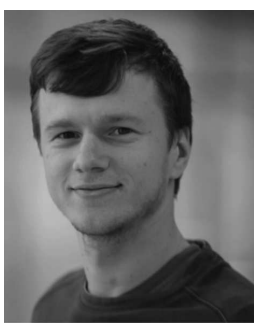

Michael Vanhoecke received the Ph.D. degree in electrical engineering from Ghent University, Ghent, Belgium, in 2019. He has been a Researcher with IDLab-Design Group, Ghent University-imec, Ghent, since 2013. He is currently a Senior Ana$\log$ ASIC Engineer with imec, Ghent. His current research interests include high-speed optoelectronic circuits and subsystems, with an emphasis on driver electronics and (segmented) Mach-Zehnder modulators for both telecom and datacom applications, as well as radiation-hardened electronics for transceivers in photonic satellite payloads.

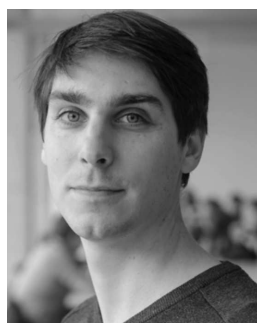

Hannes Ramon received the M.S. and Ph.D. degrees in electrical engineering from Ghent University, Ghent, Belgium, in 2015 and 2019, respectively. Since 2015, he has been working at the IDLab Design Group, Department of Information Technology, Ghent University-imec, Ghent. His research interest is in high-speed, high-frequency mixed-signal designs for (opto-) electronic communication systems.

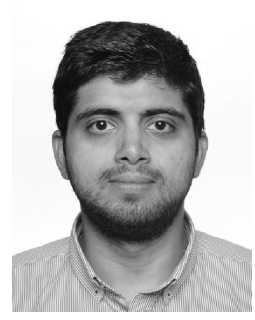

Ashwyn Srinivasan has received his $\mathrm{PhD}$ in photonics engineering from the Department of Information Technology, Ghent University, Belgium in 2018, with a focus on advanced Ge devices for optical interconnect applications. He received a master's degree in micro and nanotechnologies for integrated systems from the Swiss Federal Institute of Technology, Lausanne, Switzerland, Grenoble Institute of Technology, Grenoble, France and Politecnico di Torino, Turin, Italy in 2013 with La Mention Très Bien and 110/110 con lode. He also holds a bachelor's degree in electronics and communication engineering from the National Institute of Technology, Tiruchirappalli, India. Since 2017, he is with imec, Belgium and is a senior R\&D engineer, working on next generation transmitter and receiver concepts for optical communication and quantum optics applications. His research interests include solving complex problems in the field of optical communications, electronics, computation and quantum optics. He is a member of the Optical Society of America and IEEE Photonics Society.

Peter De Heyn received the master's degree in photonics engineering from Ghent University, Ghent, Belgium and Vrije Universiteit Brussel, Brussel, Belgium in 2009 and the Ph.D. degree from Ghent University in 2014. He has been a R\&D Engineer with IMEC, Leuven, Belgium, since 2014 and is specialized in silicon photonics design, modeling, measurements, and analysis. 


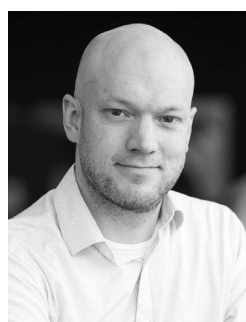

Joris Van Campenhout received the Ph.D. degree in electrical engineering from Ghent University, Ghent, Belgium, in 2007, for his work on heterogeneous integration of electrically driven InP microdisk lasers on silicon photonic waveguide circuits. He is currently a Chief Technologist in silicon photonics and the Director of the Optical I/O industry-affiliation Research and Development Program at imec, Leuven, Belgium, which covers the development of a scalable and industrially viable short-reach optical interconnect technology based on silicon photonics. Prior to joining imec in 2010, he was a Post-Doctoral Researcher with IBM's Thomas J. Watson Research Center, Ossining, NY, USA, where he developed silicon electrooptic switches for chip-level reconfigurable optical networks. He holds eight granted patents and has authored or coauthored over 100 articles in the field of silicon integrated photonics, which have received more than 7000 citations.

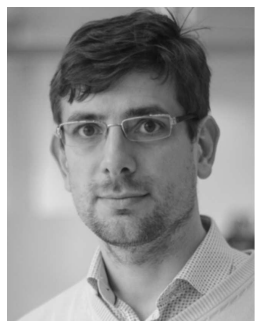

Peter Ossieur received the M.Sc. engineering degree in applied electronics and the Ph.D. in electrical engineering from Ghent University, Ghent, Belgium, in 2000 and 2005, respectively. From 2005 to 2008, he was a Postdoctoral Fellow of the Fund of Scientific Research with Ghent University. During that time, his research was focused on $10 \mathrm{Gbit} / \mathrm{s}$ burstmode receivers and optoelectronics for automotive applications. In 2008, he became a part-time Professor of High-Frequency Electronics at the Faculty of Engineering, Ghent University. In 2009, he joined the Photonic Systems Group, Tyndall National Institute and the Department of Physics, University College Cork, Cork, Ireland, where he became Senior Staff Researcher in April 2013. In October 2017, he joined imec-IDLab as a Senior Researcher and is currently Program Manager High-Speed Transceivers. He leads research activity focused on the development of highspeed analog and mixed-signal integrated circuits for photonic applications. He has (co-)authored 120 peer-reviewed papers.

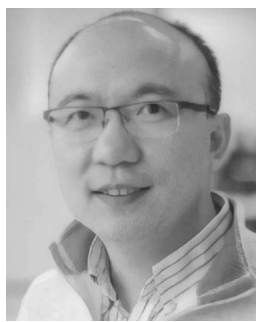

Xin Yin received the M.Sc. degree in electronics engineering from Fudan University, Shanghai, China, in 2002 and the Ph.D. degree in electronic engineering from Ghent University, Ghent, Belgium, in 2009. Since 2007, he has worked as a Staff Researcher in IMEC-INTEC and since 2013 he has been a Professor with the INTEC Department, Ghent University. He was and is active in European and International projects such as PIEMAN, EUROFOS, MARISE, C3PO, DISCUS, Phoxtrot, MIRAGE, SPIRIT, WIPE, Teraboard, STREAMS, 5GPHOS, and GreenTouch consortium. His research interests include high-speed opto-electronic circuits and transmitter/receiver subsystems, with emphasis on burst-mode receivers and clock data recovery ICs for optical access networks, and low power mixed-signal integrated circuit design for datacom/telecom/5G applications. He has authored and coauthored more than 200 journal and conference publications in the field of high-speed electronics and fiber-optic communication. In 2015, he led a team including researchers from imec, Bell Labs USA/Alcatel-Lucent and Orange Labs, which won the GreenTouch $1000 \mathrm{x}$ award in recognition of the invention of the Bi-PON protocol and sustained leadership. He has become a member of the ECOC technical program committee (TPC) since 2015.

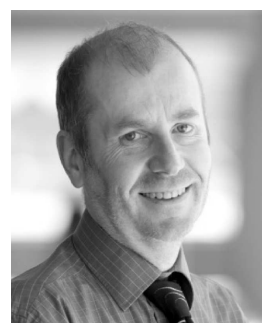

Piet Demeester is currently a Professor with Ghent University, Ghent, Belgium, and the Director of the IDLab Department, imec, Ghent. He is the coauthor of over 1000 international publications. He is the holder of an advanced ERC grant. His research activities include distributed intelligence in the Internet of Things, machine learning and data mining, semantic intelligence, cloud and big data infrastructures, fixed networking, wireless networking, electromagnetics, and high-frequency design.

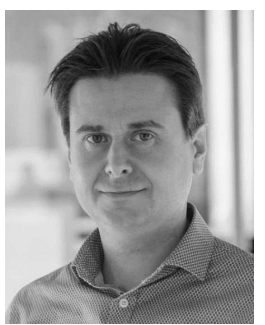

Johan Bauwelinck received the Ph.D. degree in applied sciences, electronics from the Ghent University, Ghent, Belgium, in 2005. Since October 2009, he has been a Professor with the INTEC Department at the same university and since 2014, he is leading the Internet Technology and Data Science Lab Design Group. He has promoted 18 Ph.D.s and coauthored more than 150 publications and 10 patents in the field of high-speed electronics and fiber-optic communication. He was and is active in the EU-funded projects GIANT, POWERNET, PIEMAN, EuroFOS, C3- PO, Mirage, Phoxtrot, Spirit, Flex5Gware, Teraboard, Streams and WIPE conducting research on advanced electronic integrated circuits for next generation transport, metro, access, datacenter and radio-overfiber networks. His research focuses on high-speed, high-frequency (opto-) electronic circuits and systems, and their applications on chip and board level, including transmitter and receiver analog front-ends for wireless, wired and fiber-optic communication or instrumentation systems. He is a Member of the ECOC technical program committee.

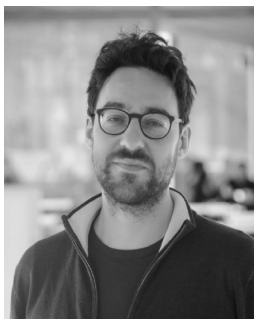

Guy Torfs received the Engineering degree in applied electronics and the Ph.D. degree in applied sciences and electronics from Ghent University, Ghent, Belgium, in 2007 and 2012, respectively. Since 2011, he has been with Ghent University-imec, Ghent, where he became an Assistant Professor in 2015. His research focuses on high-speed mixedsignal designs for wireless baseband and fiber-optic and backplane communication systems, including digital signal processing and calibration, analog equalization circuits, and clock and data recovery systems. Dr. Torfs was co-recipient of the 2015 DesignCon Best Paper Award in the High-Speed Signal Design category and the ECOC 2019 Best Demo Award. In 2014, as part of the Bi-PON and Cascaded Bi-PON Team, he was rewarded with the Greentouch 1000x Award.

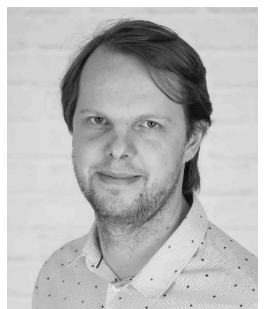

Gunther Roelkens received a degree in electrical engineering from Ghent University, Belgium, in 2002 and a $\mathrm{PhD}$ from the same university in 2007, at the Department of Information Technology (INTEC), where he is currently full professor. In 2008, he was a visiting scientist in IBM TJ Watson Research Center, New York. His research interest includes the heterogeneous integration of III-V semiconductors and other materials on top of silicon waveguide circuits and electronic/photonic co-integration. He was holder of an ERC starting grant (MIRACLE), to start up research in the field of integrated mid-infrared photonic integrated circuits. 